Also available at http://amc.imfm.si

ISSN 1855-3966 (printed edn.), ISSN 1855-3974 (electronic edn.)

ARS MATHEMATICA CONTEMPORANEA 4 (2011) 245-254

\title{
On local properties of 1-planar graphs with high minimum degree
}

\author{
Dávid Hudák， Tomáš Madaras * \\ Institute of Mathematics, Faculty of Sciences \\ University of $P$. J. Šafárik \\ Jesenná 5, 04154 Košice, Slovak Republic
}

Received 10 December 2009, accepted 23 December 2010, published online 26 May 2011

\begin{abstract}
A graph is called 1-planar if there exists its drawing in the plane such that each edge contains at most one crossing. We prove that each 1-planar graph of minimum degree 7 contains a pair of adjacent vertices of degree 7 as well as several small graphs whose vertices have small degrees; we also prove the existence of a 4-cycle with relatively small degree vertices in 1-planar graphs of minimum degree at least 6.
\end{abstract}

Keywords: 1-planar graph, light graph.

Math. Subj. Class.: 05C10

\section{Introduction}

Throughout this paper, we consider connected graphs without loops or multiple edges; we use the standard graph terminology by [8].

A graph $G$ is called planar if there exists its drawing $D(G)$ in the plane such that no two edges of $D(G)$ have an internal point (a crossing) in common; the drawing $D(G)$ with this property is called a plane graph. Planar graphs are one of the most studied graph families, possessing a wide variety of applications.

There are several different approaches generalizing the concept of planarity. One of them allows, in a drawing of a graph, a constant number of crossings per edge. In particular, if there exists a drawing $D(G)$ of a graph $G$ in the plane such that each edge of $D(G)$ contains at most one crossing, then $G$ is called 1-planar. These graphs were introduced by Ringel [19] in connection with the simultaneous vertex/face colouring of plane graphs (note 07.

* This work was supported by Science and Technology Assistance Agency under the contract No. APVV-0007-

E-mail addresses: david.hudak@student.upjs.sk (Dávid Hudák), tomas.madaras@upjs.sk (Tomáš Madaras) 
that the graph of adjacency/incidence of vertices and faces of a plane graph is 1-planar); in [19], it was proved that 1-planar graphs are 7-colourable (a linear 7-colouring algorithm was presented in [7]), and Borodin ([1,3]) proved Ringel's conjecture that these graphs are 6-colourable.

Comparing to the family of planar graphs, the family of 1-planar graphs is still only little explored (for example, except the above mentioned colouring results, there is only one other paper [5] on acyclic colourings of 1-planar graphs). In general, 1-planar graphs differ from planar ones in several fundamental aspects - they are not minor closed, their recognition is NP-complete (see [16]) and, for sufficiently large $n$, there are exponentially many nonisomorphic critical non-1-planar graphs on $n$ vertices $([15,16])$.

On the other hand, concerning the local structure, 1-planar graphs show a similar behaviour as planar graphs. Recently, the local structure of these graphs was studied in [11], [4], [12]; it was shown that, under certain conditions (like prescribed minimum degree or girth), 1-planar graphs contain various small subgraphs whose vertices have small degrees (that is, bounded above by a constant depending only on the type of the subgraph). In this paper, we continue this research by studying the local properties of 1-planar graphs which have (or are close to have) the maximum possible minimum degree (by [19], this value is equal to 7). Our motivation comes from analogical results for planar graphs of minimum degree 5 which contain a large variety of subgraphs with vertices of small degrees. For example, Borodin in [2] proved that each planar graph of minimum degree 5 contains a 3cycle with the weight (that is, the sum of degrees of its vertices) at most 17; similar results hold also for short cycles and stars (see [13, 6, 14, 17, 18]). Note that, for planar graphs of minimum degree 4, analogical results do not hold (for details, see [9] and [10]). Therefore, one may expect that, with the increasing minimum degree of a 1-planar graph, there will appear many small subgraphs with similar properties.

In the following, let $K_{2,3}^{*}$ denote a graph $K_{2,3}$ with an extra edge between two vertices of the smaller bipartition, and let $W_{5}$ denote the 5-wheel (the 4-sided pyramid graph).

We prove

\section{Theorem 1.1. Each 1-planar graph of minimum degree 7 contains}

a) a pair of adjacent 7-vertices,

b) a copy of $K_{4}$ with all vertices of degree at most 13,

c) a copy of $K_{2,3}^{*}$ with all vertices of degree at most 13 ,

d) a copy of $W_{5}$ with all vertices of degree at most 11.

In addition, we prove

Theorem 1.2. Each 1-planar graph of minimum degree 6 contains a copy of $C_{4}$ with all vertices of degree at most 47.

The proofs of all these results follow the same strategy - we assume the existence of a hypothetical counterexample $G$ to a particular theorem, and its 1-planar drawing $D=D(G)$. The drawing $D$ is then transformed into asociated plane graph $G^{\times}=$ $\left(V^{\times}, E^{\times}, F^{\times}\right)$of $G$ in such a way that all crossings of $D$ become new 4-vertices. These 4-vertices are called false, the vertices of $G^{\times}$which correspond to the original vertices of $D$ are called true; a face of $G^{\times}$is a false face if it is incident with at least one false vertex, otherwise it is a true face. Next, we proceed by Discharging Method. Each vertex and 
each face of $G^{\times}$is assigned a quantity $c$ called initial charge; this can be done in several different ways, of which we use the following two ones:

$$
\begin{aligned}
& c(v)=\operatorname{deg}_{G^{\times}}(v)-4 \text { for each vertex } v \in V^{\times}, \\
& c(\alpha)=\operatorname{deg}_{G^{\times}}(\alpha)-4 \text { for each face } \alpha \in F^{\times}, \\
& c(v)=\operatorname{deg}_{G^{\times}}(v)-6 \text { for each vertex } v \in V^{\times}, \\
& c(\alpha)=2 \operatorname{deg}_{G^{\times}}(\alpha)-6 \text { for each face } \alpha \in F^{\times},
\end{aligned}
$$

By Euler polyhedral formula, we obtain that, under the first initial charge assignment, $\sum_{x \in V^{\times} \cup F^{\times}} c(x)=-8$ (or -12 under the second assignment).

Next, the initial charges of elements of $G^{\times}$are locally redistributed in such a way that the total sum of all charges remains the same (hence, negative). This redistribution is performed by a set of discharging rules which specify the way of transfer of a charge from one element to another one in specific configurations of vertices and faces of $G^{\times}$. Finally, by a case analysis, it is shown that, after discharging, each element of $G^{\times}$has a nonnegative final charge $c^{*}$; thus, the total sum of final charges is also nonnegative, a contradiction.

For purposes of these proofs, we use more specialized notation. Given a $d$-vertex $x$ of $G^{\times}, x_{1}, \ldots, x_{d}$ will denote its neighbours in clockwise order. By $f_{i}, i=1, \ldots, d$, we denote the face of $G^{\times}$which contains the facial subwalk $x_{i} x x_{i+1}$ (index modulo $d$ ). If $f_{i}$ is a 3 -face, then $f_{i}^{\prime}$ denotes a face having the common edge $x_{i} x_{i+1}$ (index modulo $d$ ) with $f_{i}$; further, if $f_{i}^{\prime}$ is a 3 -face, then its third vertex (which is different from $x_{i}$ and $x_{i+1}$ ) will be denoted by $x_{i}^{\prime}$.

\section{Proofs}

Proof of Theorem 1.1a). By contradiction. Suppose that there exists a 1-planar graph $G$ of minimum degree 7 such that each its 7 -vertex is adjacent only with $\geq 8$-vertices. We proceed with the Discharging Method with the initial charge assignment (1); the initial charges are redistributed according to the following rules:

Rule 1: Each $\geq 7$-vertex $v \in V^{\times}$redistributes its initial charge uniformly among incident 3-faces.

Rule 2: Each 3-face with a positive charge after application of Rule 1 sends $\frac{3}{7}$ to each incident 7-vertex.

Rule 3: Each 7-vertex with a positive charge after application of Rules 1 and 2 redistributes this charge uniformly among incident false 3-faces.

We check the nonnegativity of final charges of vertices and faces of $G^{\times}$. Since false vertices and $\geq 4$-faces of $G^{\times}$are not influenced by discharging, their final charge is equal to the initial one; hence, it is nonnegative. Also, from the formulation of discharging rules, it is easy to see that the final charge of $\geq 7$-vertices is nonnegative. Thus, it is enough to analyze just the final charge of 3-faces; let $\alpha$ be a 3-face of $G^{\times}$.

Case 1: Let $\alpha$ be true and incident only with $\geq 8$-vertices. Then, by Rule $1, c^{*}(\alpha) \geq$ $-1+3 \cdot \frac{8-4}{8}=-1+\frac{3}{2}>0$.

Case 2: Let $\alpha$ be true and incident with exactly one 7-vertex. By Rules 1 and 2, $c^{*}(\alpha) \geq$ $-1+2 \cdot \frac{8-4}{8}+\frac{7-4}{7}-\frac{3}{7}=0$.

(Note that after application of Rule 1, each true 3-face has a positive charge at least $\frac{3}{7}$.) 
Case 3: Let $\alpha=[x y z]$ be false with a false vertex $x$.

Case 3.1: If both $y, z$ are $\geq 8$-vertices, then, by Rule $1, c^{*}(\alpha) \geq-1+2 \cdot \frac{8-4}{8}=-1+2 \cdot \frac{1}{2}=$ 0 .

Case 3.2: Suppose that, without loss of generality, $y$ is a 7-vertex and $z$ is an $\geq 8$-vertex. If $y$ is incident with an $\geq 4$-face, then $c^{*}(\alpha) \geq-1+\frac{7-4}{7-1}+\frac{8-4}{8}=0$; similarly, if $z$ is incident with an $\geq 4$-face, then $c^{*}(\alpha) \geq-1+\frac{7-4}{7}+\frac{8-4}{8-1}=0$. Hence, suppose that both $y, z$ are incident with 3 -faces only. Then there exists a true 3 -face incident with $y$, which sends, by Rule $2, \frac{3}{7}$ to $y$. Then, by Rule 3, $y$ sends at least $\frac{\frac{3}{7}}{7-1}=\frac{1}{14}$ to $\alpha$, and we obtain $c^{*}(\alpha) \geq-1+\frac{7-4}{7}+\frac{8-4}{8}+\frac{1}{14}=0$.

Proof of Theorem 1.1b). By contradiction. Suppose that there exists a 1-planar graph $G$ of minimum degree 7 such that each its subgraph $K_{4}$ contains at least one $\geq 14$-vertex, called big; vertices of degrees between 7 and 13 are called intermediate. We proceed with the Discharging Method with the initial charge assignment (2); the initial charges are redistributed according to following rules:

Rule 1: Each $\geq 4$-face $\alpha \in F^{\times}$redistributes its initial charge uniformly among incident 4-vertices.

Rule 2: Each intermediate vertex sends $\frac{1}{7}$ to each adjacent 4-vertex.

Rule 3: Let $[x y z]$ be a 3-face of $G^{\times}, x$ be a 4-vertex and $y$ be an intermediate vertex. Then $y$ sends additional $\frac{1}{14}$ to $x$.

Rule 4: Each big vertex sends $\frac{4}{7}$ to each adjacent 4-vertex.

Rule 5: Let $[x y z]$ be a 3-face of $G^{\times}, x$ be a 4-vertex and $y$ be a big vertex. Then $y$ sends additional $\frac{2}{7}$ to $x$.

We check the nonnegativity of final charges of vertices and faces of $G^{\times}$. From the formulation of discharging rules, it is easy to see that the final charge of all faces is nonnegative. Thus, it is enough to analyze just the final charge of vertices.

Case 1: Let $x$ be a 4-vertex of $G^{\times}$. If $x$ is incident with at least two $\geq 4$-faces, then, by Rule $1, c^{*}(x) \geq-2+2 \cdot \frac{2 \cdot 4-6}{2}=-2+2 \cdot 1=0$. If $x$ is incident with exactly one $\geq 4$-face, then, by Rules 1, 2 and 3 (or, eventually, 1, 4 and 5) we obtain the estimation $c^{*}(x) \geq-2+\frac{2 \cdot 4-6}{2}+4 \cdot \frac{1}{7}+6 \cdot \frac{1}{14}=0$. Finally, if $x$ is incident only with 3 -faces, then its neighbours in $G^{\times}$induce a $K_{4}$, hence, one of them is big; then, by Rules 2, 3, 4 and 5, we obtain $c^{*}(x) \geq-2+3 \cdot \frac{1}{7}+6 \cdot \frac{1}{14}+\frac{4}{7}+2 \cdot \frac{2}{7}=0$.

Case 2: Let $x$ be an intermediate $d$-vertex of $G^{\times}$. Then $c^{*}(x) \geq d-6-d \cdot \frac{1}{7} \geq 0$ for $d \geq 7$.

Case 3: Let $x$ be a big $d$-vertex of $G^{\times}$. Then $c^{*}(x) \geq d-6-d \cdot \frac{4}{7} \geq 0$ for $d \geq 14$.

Proof of Theorem 1.1c). By contradiction. Suppose that there exists a 1-planar graph $G$ of minimum degree 7 such that each its subgraph $K_{2,3}^{*}$ contains at least one $\geq 14$-vertex, called big; vertices of degrees between 7 and 13 are called intermediate. We proceed with the Discharging Method with the initial charge assignment (1); the initial charges are redistributed according to following rules (the first four rules are applied in the first phase of discharging): 
Rule 1: Each intermediate vertex sends $\frac{3}{7}$ to each incident 3-face.

Rule 2: Let $\alpha=[x y z]$ be a 3-face of $G^{\times}, \beta$ be an $\geq 4$-face having the common edge $x y$ with $\alpha$ and let $v$ be a true vertex of $\beta$ which is incident to $x$ or $y$. Then $v$ sends $\frac{3}{14}$ to $\alpha$.

Rule 3: Each big vertex sends $\frac{4}{7}$ to each incident 3 -face.

Rule 4: Let $\alpha=[x y z], \beta=[y z v]$ be 3-faces of $G^{\times}$and $v$ be a big vertex. Then $v$ sends $\frac{1}{7}$ to $\alpha$.

Rule 5: Each 3-face with positive charge after application of Rules 1 - 4 redistributes this charge uniformly among adjacent 3 -faces that have negative charge after application of the above-mentioned rules.

Rule 6: Each $\geq 5$-face sends $\frac{1}{5}$ to each adjacent 3 -face.

We check the nonnegativity of final charges of vertices and faces of $G^{\times}$. Firstly, all false vertices have nonnegative final charge as they are not involved in the discharging procedure. For a 4-face $\alpha, c^{*}(\alpha)=c(\alpha)=0$; if $\alpha$ is an $r$-face of $G^{\times}$with $r \geq 5$, then, by Rule 6, $c^{*}(\alpha) \geq r-4-r \cdot \frac{1}{5}=\frac{4}{5} r-4 \geq 0$.

Let $v$ be an intermediate $d$-vertex of $G^{\times}$. Observe that, in Rule 2, $v$ just further redistributes (potentially into two $\frac{3}{14}$ ) the spared charge $\frac{3}{7}$ (as Rule 1 is not applied in this situation). Hence, $c^{*}(v) \geq d-4-\frac{3}{7} d \geq 0$ for $d \geq 7$.

Let $v$ be a big $d$-vertex and $\alpha$ be a face incident with $v$. If $\alpha$ is a 3 -face, then both Rule 3 and 4 may be involved with the total contribution $\frac{4}{7}+\frac{1}{7}=\frac{5}{7}$; if $\alpha$ is an $\geq 4$-face, then Rule 2 is possibly applied with the total contribution $2 \cdot \frac{3}{14}=\frac{3}{7}$. We conclude that the maximum charge transferred from $v$ involving a face incident with $v$ is $\frac{5}{7}$; therefore, $c^{*}(v) \geq d-4-\frac{5}{7} d \geq 0$ for $d \geq 14$.

In the following, we analyze the final charge of 3 -faces in more detail.

Case 1: Let a 3-face $\alpha$ be incident with a big vertex. Then at least one of the remaining two vertices is true and, by Rules 3 and 1 or $3, c^{*}(\alpha) \geq-1+\frac{4}{7}+\frac{3}{7}=0$.

Case 2: Let all vertices of a 3-face $\alpha$ be intermediate. Then $c^{*}(\alpha) \geq-1+3 \cdot \frac{3}{7}>0$.

Case 3: Let a 3-face $\alpha$ be incident with a false vertex $c$ and two other vertices $x, y$; according to Case 1, $x, y$ are intermediate. Let $\alpha_{1}, \alpha_{2}$ and $\alpha_{3}$ be faces of $G^{\times}$incident with edges $x y, x c$ and $y c$, respectively.

Case 3.1: Let some of $\alpha_{i}, i=1,2,3$ be an $\geq 4$-face. If $\alpha_{2}$ or $\alpha_{3}$ is an $\geq 4$-face, then, by Rules 1 and $2, c^{*}(\alpha) \geq-1+2 \cdot \frac{3}{3}+\frac{3}{14}=\frac{1}{14}>0$. If $\alpha_{1}$ is an $\geq 5$-face, then, by Rules 1 and $6, c^{*}(\alpha) \geq-1+2 \cdot \frac{3}{7}+\frac{1}{5}=\frac{2}{35}>0$. Suppose that $\alpha_{1}$ is a 4 -face. Then there exists a true vertex on $\alpha_{1}$ that is incident to $x$ or $y$; hence, by Rules 2 and 1 , $c^{*}(\alpha) \geq-1+2 \cdot \frac{3}{7}+\frac{3}{14}=\frac{1}{14}>0$.

Case 3.2: Let all $\alpha_{i}, i=1,2,3$, be 3-faces. Denote $\alpha_{1}=\left[x y z_{1}\right], \alpha_{2}=\left[x c z_{2}\right], \alpha_{3}=\left[y c z_{3}\right]$ (observe that $z_{2}$ and $z_{3}$ are true vertices).

Case 3.2.1: Let $z_{1}$ be a true vertex. Then at least one of $z_{1}, z_{2}, z_{3}$ is big (to avoid a light copy of $\left.K_{2,3}^{*}\right)$; by Rules 1 and 4, we have $c^{*}(\alpha) \geq-1+2 \cdot \frac{3}{7}+\frac{1}{7}=0$.

Case 3.2.2: Suppose that $z_{1}$ is false; further, we can suppose that both $z_{2}, z_{3}$ are intermediate (otherwise we argue similarly as in the Case 3.2.1). Let $\beta_{1}, \beta_{2}$ be faces that are adjacent to $\alpha_{1}$ through the edge $x z_{1}$ and $y z_{1}$, respectively. If both $\beta_{1}, \beta_{2}$ are $\geq 4$-faces, then, by Rules 1 and 2, the face $\alpha_{1}$ receives at least $2 \cdot \frac{3}{7}+2 \cdot \frac{3}{14}=\frac{9}{7}$ and (having initial charge -1 ) keeps at least $\frac{2}{7}$. Since $\alpha$ is the only 3-face adjacent to $\alpha_{1}$, the face $\alpha_{1}$ sends at least 
$\frac{2}{7}$ to $\alpha$ by Rule 5 , so that $c^{*}(\alpha) \geq-1+2 \cdot \frac{3}{7}+\frac{2}{7}>0$. If exactly one of $\beta_{1}, \beta_{2}$, say $\beta_{1}$, is a 3-face, then its third vertex (different from $x, z_{1}$ ) is big (otherwise we find a light copy of $K_{2,3}^{*}$ ). Using Rules 1,4 and 2 (note that $z_{1}$ is adjacent to a true vertex incident with $\beta_{2}$ ), we obtain that $\alpha_{1}$ receives at least $2 \cdot \frac{3}{7}+\frac{1}{7}+\frac{3}{14}=\frac{17}{14}$ and (subtracting the initial charge -1 ) keeps at least $\frac{3}{14}$. By Rule 5, this charge is sent to $\alpha$, and so we obtain $c^{*}(\alpha) \geq-1+2 \cdot \frac{3}{7}+\frac{3}{14}>0$. Finally, suppose that both $\beta_{1}, \beta_{2}$ are 3 -faces. Then both these faces are incident with big vertices and, by Rules 1 and 3, they receive at least $\frac{3}{7}+\frac{4}{7}=1$; we conclude that, after the first phase, $\beta_{1}$ and $\beta_{2}$ have nonnegative charge (hence, by Rule 5, $\alpha_{1}$ sends a charge to at most one 3-face). Using Rules 1 and 4, we obtain that, in the first phase, $\alpha_{1}$ receives at least $2 \cdot \frac{3}{7}+2 \cdot \frac{1}{7}$; thus, by Rule 5, $\alpha_{1}$ may send at least $\frac{1}{7}$ to $\alpha$ and we have $c^{*}(\alpha) \geq-1+2 \cdot \frac{3}{7}+\frac{1}{7}=0$.

Proof of Theorem 1.1d). By contradiction. Suppose that there exists a 1-planar graph $G$ of minimum degree 7 such that each its subgraph $W_{5}$ contains a big vertex of degree at least 12 . We proceed with the Discharging Method with the initial charge assignment (1); the initial charges are redistributed according to following rules (which are applied sequentially; by $c_{i}(x)$, we denote the charge of an element $x \in V^{\times} \cup F^{\times}$after application of the first $i$ rules):

Rule 1: Each $\geq 7$-vertex sends $\frac{4}{9}$ to each incident false 3 -face and $\frac{1}{3}$ to each incident true 3-face.

Let $k(v)$ be the number of false 3-faces incident with a vertex $v$.

Rule 2: If $v$ is a vertex of $G^{\times}$with $c_{1}(v) \geq \frac{1}{18} k(v)$, then $v$ sends $\frac{1}{18}$ to each incident false 3 -face; if $c_{1}(v)<\frac{1}{18} k(v)$ or $k(v)=0$, no charge is transferred.

Let $n(v)$ be the number of 3-faces $[x y z]$ such that $x$ is a 7-vertex and $y$ is a false vertex of $G^{\times}$(such faces will be called awkward).

Rule 3: If $v$ is a non-big vertex of $G^{\times}$with $c_{2}(v)>0$, then $v$ shares its charge equally among all incident awkward faces; further, if $c_{2}(v) \geq \frac{1}{18} n(v)$, then $v$ sends $\frac{1}{18}$ to each incident awkward face.

Rule 4: Each big vertex sends $\frac{1}{3}$ to each adjacent 7-vertex.

Rule 5: If a 7-vertex $v$ has received a contribution from a big vertex, then this contribution is further redistributed equally among all false 3-faces incident with $v$.

We check the nonnegativity of final charges of vertices and faces of $G^{\times}$. As $\geq 4$-faces and 4-vertices are not influenced by the discharging, it is enough to analyze 3-faces and $\geq 7$-vertices of $G^{\times}$.

Case 1: Let $\alpha=[x y z]$ be a 3-face.

Case 1.1: If $\alpha$ is a true 3-face, then, by Rule $1, c^{*}(\alpha)=c_{1}(\alpha) \geq-1+3 \cdot \frac{1}{3}=0$.

Case 1.2: Suppose that $\alpha$ is a non-awkward false 3-face and $z$ is its false vertex. Let $x$ be a $d$-vertex, $d \geq 8$. Then $c_{1}(x) \geq d-4-d \cdot \frac{4}{9}=\frac{5}{9} d-4 \geq \frac{1}{18} d$. Thus $c_{1}(x) \geq \frac{1}{18} k(x)$ and, by Rule 2, $x$ sends $\frac{1}{18}$ to $\alpha$. The same consideration applies to $y$; hence, we obtain that $c^{*}(\alpha) \geq-1+2 \cdot \frac{4}{9}+2 \cdot \frac{1}{18}=0$.

Case 1.3: Let $\alpha$ be an awkward face with 7-vertex $x$. 
Case 1.3.1: Let $y$ be a big $d$-vertex. Then $c_{1}(y) \geq d-4-\frac{4}{9} d=\frac{5}{9} d-4 \geq \frac{1}{18} d \geq \frac{1}{18} k(y)$; hence, by Rule 2, $y$ sends $\frac{1}{18}$ to $\alpha$. Further, $x$ receives $\frac{1}{3}$ from $y$ by Rule 4 . By parity argument, $k(x) \leq 6$, and $x$ sends, by Rule 5 , at least $\frac{1}{6} \cdot \frac{1}{3}=\frac{1}{18}$ to each incident false 3 -face. Therefore, we obtain $c^{*}(\alpha) \geq-1+2 \cdot \frac{4}{9}+\frac{1}{18}+\frac{1}{18}=0$.

Case 1.3.2: Let $y$ be a $d$-vertex with $d \in\{9,10,11\}$. Note that $c_{1}(y) \geq \frac{1}{18} k(y)$ and $y$ sends $\frac{1}{18}$ to $\alpha$ by Rule 2. Further, $c_{2}(y) \geq d-4-k(y) \cdot \frac{4}{9}-(d-k(y)) \cdot \frac{1}{3}-n(y) \cdot \frac{1}{18}=$ $\frac{2}{3} d-4-\frac{1}{9} k(y)-\frac{1}{18} n(y) \geq \frac{2}{3} d-4-\frac{1}{9} k(y)-\frac{1}{18} k(y)=\frac{2}{3} d-4-\frac{3}{18} k(y) \geq \frac{2}{3} d-4-\frac{3}{18} d=$ $\frac{9}{18} d-4 \geq \frac{1}{18} d$ for $d \geq 9$. This implies that $y$ sends additional $\frac{1}{18}$ to $\alpha$ by Rule 3 ; in total, we obtain $c^{*}(\alpha) \geq-1+2 \cdot \frac{4}{9}+\frac{1}{18}+\frac{1}{18}=0$.

Case 1.3.3: Let $y$ be an 8 -vertex. Still, $y$ sends $\frac{1}{18}$ to $\alpha$ by Rule 2. Suppose first that $x$ is incident with at least one $\geq 4$-face. Then $c_{1}(x) \geq 7-4-6 \cdot \frac{4}{9}=\frac{3}{9}=\frac{1}{18} \cdot 6$; hence, $x$ may send $\frac{1}{18}$ to $\alpha$ by Rule 2 . Now, let $x$ be incident only with 3 -faces; note that, in this case, the number of false 3-faces containing $x$ is even. If at least three of them are true 3 -faces, then $c_{1}(x) \geq 7-4-4 \cdot \frac{4}{9}-3 \cdot \frac{1}{3}=\frac{2}{9}=(7-3) \cdot \frac{1}{18}$ and again, $x$ can send $\frac{1}{18}$ to $\alpha$ by Rule 2 . So suppose that there is exactly one true 3 -face incident with $x$. Then $x$ and its four true neighbours induce a copy of $W_{5}$ in $G$. Thus, at least one of neighbours of $x$ is big and sends, by Rule $4, \frac{1}{3}$ to $x$; by Rule 5 , this charge is equally redistributed among six false 3 -faces incident with $x$, which yields the contribution $\frac{1}{18}$ to $\alpha$, and so $c^{*}(\alpha) \geq-1+2 \cdot \frac{4}{9}+\frac{1}{18}+\frac{1}{18}=0$.

Case 1.3.4: Let $y$ be a 7-vertex. Then we may use the arguments from Case 1.3.3 concluding that each of $x, y$ sends $\frac{1}{18}$ to $\alpha$ either by Rule 2 or by Rule 5. Hence, we have $c^{*}(\alpha) \geq-1+2 \cdot \frac{4}{9}+2 \cdot \frac{1}{18}=0$.

Case 2: Let $x$ be a non-big $d$-vertex. Note that transfers from $x$ by Rules 2, 3 or 5 are performed only if $x$ has sufficient charge after application of Rule 1; after these subsequent transfers, $x$ is discharged to 0 . Hence, it is enough to check the situation after using the Rule 1. Let $t$ be number of transfers of $\frac{4}{9}$ by Rule 1 from $x$ to incident false 3 -faces, and $q$ the number of transfers of $\frac{1}{3}$ from $x$ to other incident 3-faces. Then $t+q \leq d$ and $t \leq 2 \cdot\left\lfloor\frac{d}{2}\right\rfloor$, and we obtain $c_{1}(x) \geq d-4-\frac{4}{9} t-\frac{1}{3} q=d-4-\frac{1}{3}(t+q)-\frac{1}{9} t \geq d-4-\frac{1}{3} d-\frac{1}{9} \cdot 2 \cdot\left\lfloor\frac{d}{2}\right\rfloor \geq 0$ for $d \geq 7$.

Case 3: Let $x$ be a big $d$-vertex. Let $n$ and $p$ be numbers of false and true 3 -faces incident with $x$ and $s$ be the number of 7-vertices adjacent to $x$. Then $p+n \leq d$ and $s \leq d$; moreover, since each pair of false 3-faces in the neighbourhood of $x$ contains at least one vertex of degree $\neq 7$, it follows that $p+n+s \leq 2 d-\frac{n}{2}$. Then, by Rules 1,2 and 4 , we obtain the estimation $c^{*}(x) \geq d-4-\frac{4}{9} n-\frac{1}{3} p-\frac{1}{18} n-\frac{1}{3} s=d-4-\frac{n}{2}-\frac{p}{3}-\frac{s}{3} \geq$ $d-4-\frac{2}{3} d=\frac{d}{3}-4 \geq 0$ for $d \geq 12$.

Proof of Theorem 1.2. By contradiction. Suppose that there exists a 1-planar graph $G$ of minimum degree 6 such that each its 4-cycle contains a big vertex of degree at least 48 . We proceed with the Discharging Method with the initial charge assignment (2); the initial charges are redistributed according to the following rules :

Rule 1: Each big face sends $\frac{1}{2}$ to each incident 4-vertex.

Rule 2: Let $\beta=[x y z]$ be a 3-face having the common edge $y z$ with a big face $\alpha$, and let $x$ be a 4 -vertex. Then $\alpha$ sends $\frac{1}{4}$ to $x$. 
Rule 3: Let $\beta=[x y z]$ be a 3-face having the common edge $x y$ with a big face $\alpha$, and let $x$ be a 4 -vertex. Then $\alpha$ sends $\frac{1}{8}$ to $x$.

Rule 4: Let $\beta=[x y z]$ be a 3-face adjacent with a 3-face $\gamma=[y z w]$ that has a common edge $z w$ with a big face $\alpha$, and let $x$ be a 4 -vertex. Then $\alpha$ sends $\frac{1}{8}$ to $x$.

Rule 5: Let $\alpha, \beta$ be two incident big faces with the common edge $x y$, and let $x$ be a 4 -vertex. Then $\alpha$ sends $\frac{1}{4}$ to $x$.

Rule 6: Let $y$ be a big vertex adjacent to an 4-vertex $x$. Then $y$ sends $\frac{5}{8}$ to $x$.

Rule 7: Let $\alpha=[x y z]$ be a 3-face, $y$ be big, and $x$ be a 4 -vertex. Then $y$ sends additional $\frac{5}{16}$ to $x$.

Rule 8: Let $\alpha=[x y z], \beta=[y z w]$ be 3-faces, $w$ be big, and $x$ be a 4 -vertex. Then $w$ sends $\frac{1}{4}$ to $x$.

Rule 9: Let $\alpha=[x y z], \beta=[x y w]$ be 3 -faces, $w$ be big, and $x$ be a 4 -vertex. Then $w$ sends additional $\frac{1}{8}$ to $x$.

Rule 10: Let $\alpha=[x y z]$ be a 3-face having a common edge $x y$ with a big face $\beta, z$ be big, and $x$ be a 4 -vertex. Then $z$ sends additional $\frac{1}{4}$ to $x$.

Rule 11: Let $\alpha=[x y z], \beta=[y z v]$, and $\gamma=[y v w]$ be 3-faces, $w$ be big, and $x$ be a 4-vertex. Then $w$ sends additonal $\frac{1}{8}$ to $x$.

We will analyze the final charges of vertices and faces of $G^{\times}$.

Case 1: Let $x$ be a 4 -vertex.

Case 1.1: If all $f_{i}, i=1, \ldots, 4$ are big, then, by Rule $1, c^{*}(x) \geq-2+4 \cdot \frac{1}{2}=0$.

Case 1.2: Let exactly one of $f_{i}$, say $f_{4}$, be a 3 -face. Then $c^{*}(x) \geq-2+3 \cdot \frac{1}{2}+2 \cdot \frac{1}{8}+4 \cdot \frac{1}{4}=$ $\frac{3}{4}>0$ by Rules 1 (applied three times), 3 (twice), and 5 (four times).

Case 1.3: Let two of $f_{i}, i=1, \ldots, 4$ be 3 -faces. According to the symmetry, we distinguish two cases:

Case 1.3.1: Let $f_{2}, f_{4}$ be 3 -faces. Then one of $x_{i}$, say $x_{1}$, is big (otherwise $x_{1} x_{3} x_{2} x_{4}$ is a light 4 -cycle). By Rules 1 (twice), 3 (four times), 6 (once) and 7 (once), $c^{*}(x) \geq$ $-2+2 \cdot \frac{1}{2}+4 \cdot \frac{1}{8}+\frac{5}{8}+\frac{5}{16}>0$.

Case 1.3.2: Let $f_{1}, f_{4}$ be 3 -faces. If one of $x_{1}, \ldots, x_{4}$ is big, then $c^{*}(x) \geq-2+2 \cdot \frac{1}{2}+2$. $\frac{1}{8}+2 \cdot \frac{1}{4}+\frac{5}{8}>0$ by Rules 1 (applied twice), 3 (twice), 5 (twice), and 6 (once).

So, assume that no neighbour of $x$ is big. Consider the face $f_{1}^{\prime}$. If $f_{1}^{\prime}$ is big, then it sends $\frac{1}{4}$ to $x$ by Rule 2 ; otherwise it is a 3 -face. If $x_{1}^{\prime}$ is a true vertex, then it must be big (otherwise $x_{1} x_{1}^{\prime} x_{2} x_{4}$ is a light 4 -cycle). The big vertex $x_{1}^{\prime}$ sends $\frac{1}{4}$ to $x$ by Rule 8 . It remains to resolve the case when $f_{1}^{\prime}$ is a 3 -face, but $x_{1}^{\prime}$ is a false vertex. Then consider the faces $f_{1}^{\prime l}, f_{1}^{\prime r}$ that share with $f_{1}^{\prime}$ common edge $x_{1}^{\prime} x_{1}$ or $x_{1}^{\prime} x_{2}$, respectively. If some of them is a 3 -face, then its third vertex $z \neq x_{1}^{\prime}, x_{1}$ (or $z \neq x_{1}^{\prime}, x_{2}$ ) must be big (otherwise $x_{4} x_{1} z x_{2}$ is a light 4 -cycle). Hence, it contributes $\frac{1}{8}$ to $x$ by Rule 11 . If some of these faces is big, then it sends $\frac{1}{8}$ to $x$ by Rule 4 . Consequently, $x$ receives $\geq \frac{1}{4}$ due to the transfers through edge $x_{1} x_{2}$. The same consideration is applied on the transfers through edge $x_{4} x_{1}$. Thus, we obtain $c^{*}(x) \geq-2+2 \cdot \frac{1}{2}+2 \frac{1}{8}+2 \cdot \frac{1}{4}+2 \cdot \frac{1}{4}=\frac{1}{4}>0$.

Case 1.4: Let three of $f_{i}$, say $f_{1}, f_{2}$, and $f_{4}$, be 3 -faces. Note that at least one of neighbours of $x$ is big.

Case 1.4.1: If $x$ has at least two big neighbours, then $c^{*}(x) \geq-2+\frac{1}{2}+2 \cdot \frac{1}{8}+2 \cdot \frac{5}{8}+2$. $\frac{5}{16}+2 \cdot \frac{1}{8}>0$ by Rules 1 (applied once), 3 (twice), 6 (at least twice), 7 (at least twice) and 9 (at least twice). 
Case 1.4.2: Let $x$ have exactly one big neighbour. If $x_{1}$ or $x_{2}$ is big, then $c^{*}(x) \geq-2+$ $\frac{1}{2}+2 \cdot \frac{1}{8}+\frac{5}{8}+2 \cdot \frac{5}{16}+\frac{1}{8}+\frac{1}{4}>0$ by Rules 1 (applied once), 3 (twice), 6 (once), 7 (twice), 9 (once) and 10 (once).

Suppose, without loss of generality, that $x_{3}$ is big. Consider now the faces $f_{1}^{\prime}, f_{4}^{\prime}$. We may use the same argumentation as in Case 1.3.2. The transfer through edges $x_{1} x_{2}$ and $x_{1} x_{4}$ is always at least $2 \cdot \frac{1}{4}$. Thus, in total, $c^{*}(x) \geq-2+\frac{1}{2}+2 \cdot \frac{1}{8}+\frac{5}{8}+\frac{5}{16}+\frac{1}{8}+2 \cdot \frac{1}{4}>0$ by Rules 1 (applied once), 3 (twice), 6 (once), 7 (once), 9 (once), and the above mentioned contribution.

Case 1.5: Let all faces incident with $x$ be 3 -faces. Note that at least one of neighbours of $x$ is big. If at least two neighbours of $x$ are big, then $c^{*}(x) \geq-2+2 \cdot \frac{5}{8}+4 \cdot \frac{5}{16}+2 \cdot \frac{1}{8}>0$ by Rules 6 (twice), 7 (four times) and 9 (four times). Let exactly one of neighbours of $x$, say $x_{1}$, be big. Then, using the same argumentation as in Case 1.3.2, $x$ receives at least $2 \cdot \frac{1}{4}$ through edges $x_{2} x_{3}$ and $x_{3} x_{4}$. In total, $c^{*}(x) \geq-2+\frac{5}{8}+2 \cdot \frac{5}{16}+2 \cdot \frac{1}{8}+\frac{1}{2}=0$ by Rules 6 (applied once), 7 (twice), 9 (twice), and the above mentioned contribution.

Case 2: Let $\alpha$ be a big face. Observe that Rules 3,4 just halve the charge $\frac{1}{4}$ that is saved (since, in situation of Rules 3,4, there is no such transfer as in Rule 2). Also, in Rule 5, the full transfer of $\frac{1}{4}$ is saved, because the situation of Rules 3 and 4 is not involved in this rule. Hence, we may roughly consider that, through each edge incident with $\alpha, \frac{1}{4}$ of charge is transfered. Also, note that Rule 1 applies to at most $\left\lfloor\frac{\operatorname{deg}(\alpha)}{2}\right\rfloor$ vertices incident with $\alpha$. Therefore, $c^{*}(\alpha) \geq c(\alpha)-\left\lfloor\frac{\operatorname{deg}(\alpha)}{2}\right\rfloor \cdot \frac{1}{2}-\operatorname{deg}(\alpha) \cdot \frac{1}{4} \geq 2 \cdot \operatorname{deg}(\alpha)-6-\frac{\operatorname{deg}(\alpha)}{4}-\frac{\operatorname{deg}(\alpha)}{4}=$ $\frac{3}{2} \cdot \operatorname{deg}(\alpha)-6 \geq 0$ if $\operatorname{deg}(\alpha) \geq 4$.

Case 3: Let $x$ be a big vertex. Note that Rule 7 just halves the charge that is saved in the corresponding situation when Rule 6 is not used, Rules 9,11 work in the same way in connection with Rule 8 . The Rule 10 sends the full charge that was saved in Rule 8 . We can use very rough estimation $\frac{5}{8}+\frac{1}{4}$ for a charge assigned to a neighbour of $x$, and to an edge of a 3 -face incident with $x$. By this rough estimation, $c^{*}(x) \geq c(x)-\left(\frac{5}{8}+\frac{1}{4}\right) \cdot \operatorname{deg}(x)=$ $\operatorname{deg}(x)-6-\frac{7}{8} \cdot \operatorname{deg}(x)=\frac{1}{8} \cdot \operatorname{deg}(x)-6 \geq 0$ for $\operatorname{deg}(x) \geq 48$.

\section{References}

[1] O. V. Borodin, Solution of the Ringel problems on the vertex-face coloring of plane graphs and the coloring of 1-planar graphs, Metody Diskretn. Anal. 41 (1984), 12-26.

[2] O. V. Borodin, Solution of Kotzig-Grünbaum problems on separation of a cycle in planar graphs, Mat. Zametki 46 (1989), 9-12, (in Russian).

[3] O. V. Borodin, A new proof of 6 color theorem, J. Graph Theory 19 (1995), 507-521.

[4] O. V. Borodin, I. G. Dmitriev and A. O. Ivanova, The height of a cycle in 1-planar graphs with minimum degree 5 without triangles, Diskretn. Anal. Issled. Oper. 15 (2008), 11-16, (in Russian).

[5] O. V. Borodin, A. V. Kostochka, A. Raspaud and E. Sopena, Acyclic colouring of 1-planar graphs, Discrete Appl. Math. 114 (2001), 29-41.

[6] O. V. Borodin and D. Woodall, Short cycles of low weight in normal plane maps with minimum degree 5, Discussiones Math. Graph Th. 16 (1998), 159-164.

[7] Z.-Z. Chen and M. Kouno, A linear-time algorithm for 7-coloring 1-plane graphs, Algorithmica 43 (2005), 147-177. 
[8] R. Diestel, Graph theory, Springer 2006.

[9] I. Fabrici, E. Hexel, S. Jendrol' and H. Walther, On vertex-degree restricted paths in polyhedral graphs, Discrete Math. 212 (2000), 61-73.

[10] I. Fabrici and S. Jendrol', Subgraphs with restricted degrees of their vertices in planar 3connected graphs, Graphs and Combin. 13 (1997), 245-250.

[11] I. Fabrici and T. Madaras, The structure of 1-planar graphs, Discrete Math. 307 (2007), 854865.

[12] D. Hudák and T. Madaras, On local structure of 1-planar graphs of minimum degree 5 and girth 4, Discussiones Math. Graph. Th. 29 (2009), 385-400.

[13] S. Jendrol' and T. Madaras, On light subgraphs in plane graphs of minimum degree five, Discussiones Math. Graph Th. 16 (1996), 207-217.

[14] S. Jendrol', T. Madaras, R. Soták and Z. Tuza, On light cycles in plane triangulations, Discrete Math. 197/198 (1999), 453-467.

[15] V. P. Korzhik, Minimal non-1-planar graphs, Discrete Math. 308 No. 7 (2008), 1319-1327.

[16] V. P. Korzhik and B. Mohar, Minimal obstructions for 1-immersions and hardness of 1planarity testing, Springer Lecture Notes in Computer Science 5417 (2009), 302-312.

[17] T. Madaras, R. Škrekovski and H.-J. Voss, The 7-cycle $C_{7}$ is light in the family of all plane graphs with minimum degree 5, Discrete Math. 307 (2007), 1430-1435.

[18] B. Mohar, R. Škrekovski and H.-J. Voss, Light subgraphs in planar graphs of minimum degree 4 and edge degree 9, J. Graph Theory 44 (2003), 261-295.

[19] G. Ringel, Ein Sechsfarbenproblem auf der Kugel, Abh. Math. Sem. Univ. Hamburg 29 (1965), 107-117. 